\title{
Food Systems Research to Support Sustainable Impact
}

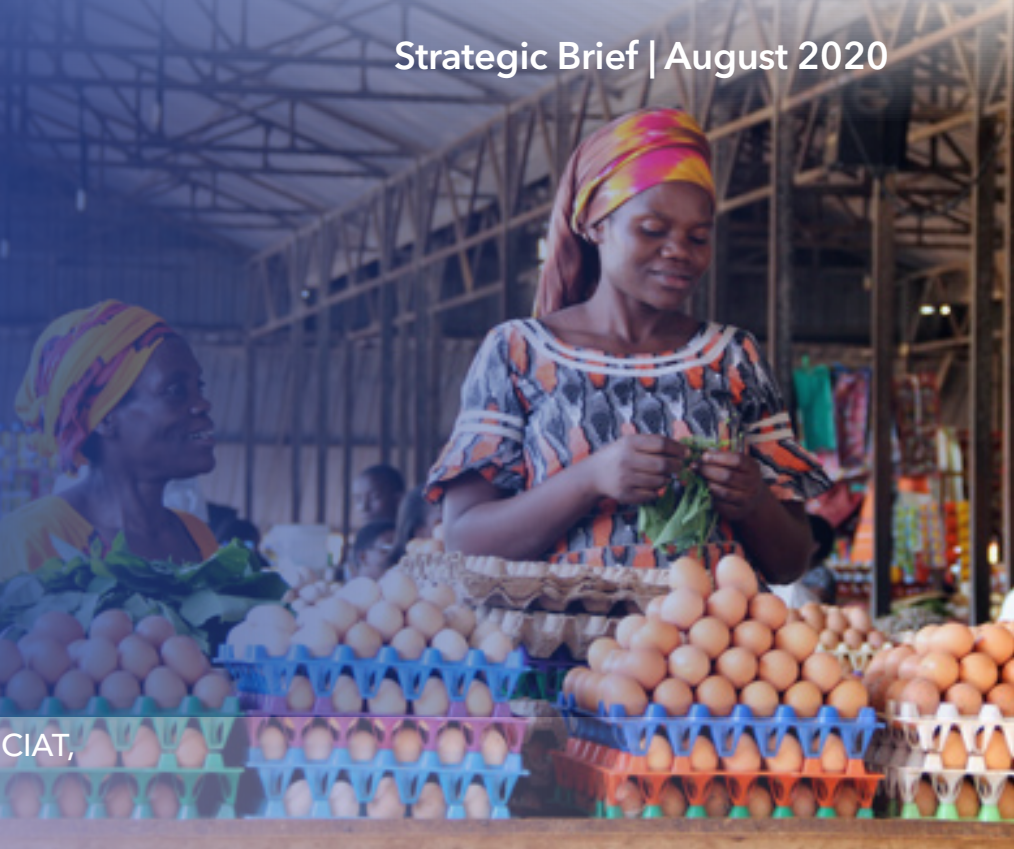

Chris Béné and Mark Lundy, Alliance of Bioversity International and CIAT, Alan de Brauw, International Food Policy Research Institute, and Inge D. Brouwer, Wageningen University \& Research

Food systems, which are essential sources of food, but also of income and employment, especially for resource-poor populations in low- and middle-income countries (LMICs), are undergoing dramatic transformations. Driven by changes in diets, urbanization, longer supply chains, and new ways of purchasing and consuming foods, these transformations pose serious challenges to achieving healthy, environmentally sustainable, and inclusive food systems for all. Rates of undernutrition and micronutrient deficiencies are still stubbornly high, while overweight, obesity, and accompanying noncommunicable diseases are rising - with these multiple forms of malnutrition increasingly found within the same communities, households, or even individuals.

Diets increasingly include ultra-processed, nutrient-poor foods that lure consumers with convenience, price, and taste. Growing pressures are put on the natural environment as agricultural production intensifies to meet rising national and global demand. The distance between where food is grown and where it is eaten continues to widen, adding significant environmental costs and food safety risks. Food loss and waste, especially of nutritious but perishable fruits, vegetables, and animal-sourced foods, but also for cereals and pulses, threaten system efficiency, food and nutrition security, and environmental sustainability. Meanwhile, healthy diets are not affordable for much of the world's low-income population concentrated in LMICs.

Countries need support across the different domains of these transitions, with research, data, and the opportunity to learn from each other's experiences, to ensure national food systems transform in a way that provides more sustainable access to healthy and safe diets for all. The CGIAR Research Program on Agriculture for Nutrition and Health (A4NH) has coordinated and led CGIAR efforts to meet these needs. The work

\section{Key Messages on Food Systems Research}

- Starting with consumers and food environments will help research to address rapid food system changes that promote healthier diets for better nutrition and health outcomes, environmental sustainability, economic development, and social equity and inclusiveness.

- Evidence is being built, using systems research, for relevant, context-specific entry points relevant to addressing national goals ranging from agriculture to transport, processing to marketing, sales to consumer choice, and food preparation to consumption.

- Research should focus on food system dynamics and highlight potential interactions, including synergies and trade-offs, between drivers, components, stakeholders, and outcomes that go beyond simple short-term fixes.

- The most challenging task is to support food governance with a systemic perspective - not considering just one food or one instrument, but acknowledging its multiscale nature, from local to global.

- A country presence in national policy and program dialogue for alignment to national goals is essential.

breaks down into four focus areas for food systems research to support the following national food system policy and program developments:

1. Reverse Thinking: Putting Diets First: Instead of only starting from policies and innovations that attempt to shape food production, it is critical to consider the diet from the perspective of consumer demand. 
FIGURE 1: Conceptual framework of food systems for diets and nutrition

\section{Drivers}

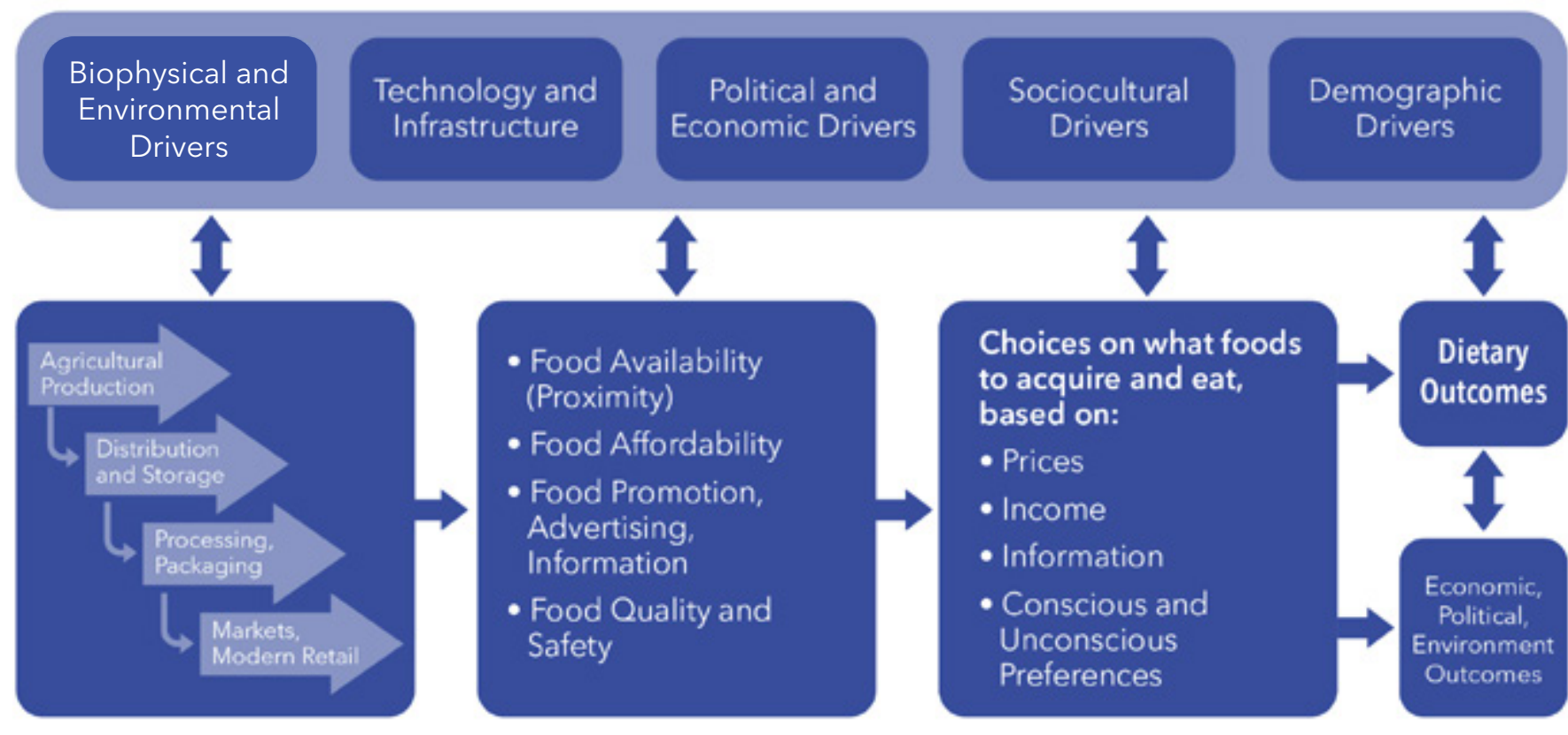

Food Value Chains

Food Environment

\section{Consumer Behavior}

Source: From de Brauw et al, 2019

2. Food System Innovations: For the desired transformations to occur, countries must harness the combined power and potential of innovations in technology, infrastructure, and institutions to foster sustainable food system developments that promote healthy diets.

3. Enabling Environment: An enabling food environment calls for designing and adapting food systems policies while recognizing potential trade-offs and reducing unintended consequences. This requires participation from the private sector for market opportunities that support food systems transformation in a positive way.

4. Ensure No One is Left Behind: Poor people, those living in rural and peri-urban areas, ethnic minorities, youth, and women are just some examples of groups frequently left behind. Policymakers should look for inclusive growth strategies and opportunities for everyone to benefit from improved diets and market opportunities created as food systems transform.

While definitions of food systems vary, the following key components are generally accepted (Figure 1): (1) drivers: external factors like urbanization, technology development, climate change, and economic growth; (2) components: including food production, distribution, allocation, and the public and private food environment; and (3) outcomes: related to healthy diets, environmental sustainability, economic development, and social equity and inclusiveness. Very little is known about potential interactions (both synergies and trade-offs) between drivers, components, and their outcomes. Until recently, most international reports' food systems analysis focused primarily on improving food production efficiency under different conditions of population growth and climate change or on improving markets and institutions to reduce transaction costs and risks. Very few have emphasized demand for food or conditions for guaranteeing access to healthy diets. Even fewer studies use a systems perspective or explore governance for a responsive and adaptive food environment, in spite of trade-offs between different food system components being increasingly registered and needing to be explicitly navigated when developing food system innovations. There is no real consensus on how to make transformations operational (beyond simple technical fixes) or give attention to changes in interactions between stakeholders and adjustments in food system dynamics. Systems research can help build evidence for relevant entry points from agriculture to transport, processing to marketing, sales to consumer choices, and preparation to consumption.

\section{Progress Toward Food Systems for Healthier Diets}

CGIAR food systems research, through A4NH's Food Systems for Healthier Diets (FSHD) flagship, moves along a deliberate trajectory from diagnosis and foresight assessments of national and subnational drivers of food system transformation, to testing concrete innovations and interventions, to scaling up and anchoring successful actions, in collaboration with relevant 
In the last few years, several policy regulations and experiments have been implemented that try to reduce the negative effects of the rapid changes observed in modern food systems, with some promising results. In Mexico, Thailand, and Chile, for instance, sugary drink taxes and front-of-the-pack warning labels have been used with some success to influence reformulation of unhealthy food products by the private sector, as well as to discourage the purchase of such foods. Social protection programs, when combined with behavior change communication, have been shown to substantially improve nutrition outcomes in low-income households. Finally, recent experience shows that, when it comes to interventions, city-region is often the more effective scale.

While engaging in the sphere of food systems policy or trying to guide/advise decision-makers about "what needs to be done to improve food systems," decisions have to be made now; one cannot hide behind the lack of data or the difficulty of embracing a multisectoral, multi-actor, multi-scale vision to act. Food systems are among the major sources of environmental, health, and social externalities; the COVID-19 pandemic is a vivid illustration of this reality.

It is vital to also engage and build capacity among stakeholders at the national level to act toward building food systems that support healthier diets. In FSHD, this has been done at different levels: involving national-level professionals to develop foodbased dietary guidelines in Ethiopia, retailers to increase fruit and vegetable intake in Viet Nam and Nigeria, and also eager young scientists at the MSc and PhD level from the focal countries to help develop future leaders in all four countries.

\section{Next Steps and Further Research Priorities}

Against this backdrop of on-going work, several key areas for future research emerge:

\section{- Greater understanding of consumers and food consump-}

tion choices and the relationships with existing and changing food environments. It is critical to know what drives consumption decisions toward healthy or unhealthy choices; how availability, affordability, consumer messaging, and acceptability influence those decisions and what effective mechanisms or policy instruments exist or should be developed to support informed and healthier choices by consumers in different contexts.

- Understanding the role of private actors and market systems in food provision, processing, and distribution from farmgate to the consumer plate by identifying robust approaches to improve the delivery/availability of key food groups such as vegetables, fruits, milk, eggs, fish, and meat while guaranteeing food safety, reduced spoilage, and efficiency. Strategies to engage the local, national, and global food industry are needed to improve nutritional density of processed and prepared foods, increase micronutrient availability, eliminate aflatoxins, and proactively enter into product reformulation aligned with the population's nutrition needs. Ultimately, circular food economies employing ICT solutions, can be helpful to improve traceability, reduce food loss and waste, improve nutrient cycling from consumption back to production, and increase consumer confidence in the quality of their foods.

- Situating and analyzing these components in specific contexts with local system actors to understand interactions, governance, and outcomes. This includes unpacking drivers to understand food system transitions across countries and along a national and subnational urban-rural continuum, constructing tools and approaches to systematically assess environmental, economic, and nutrition performance, and assisting national and subnational actors in designing and testing interventions to better achieve their priority outcomes.

\section{- Co-designing and co-developing effective tools and pro-} cesses to take the pulse of the food system, identify options, analyze trade-offs between multiple food system outcomes related to economic, environmental, and health goals, design interventions, and evaluate outcomes with national and subnational actors.

- Develop explicit institutional arrangements to accelerate learning (platforms, networks).

- Finally, to support the needed country engagement for food systems research calls for a strong country presence in national policy and program dialogues to identify helpful research entry points synergistic to delivering on national goals.
INTERNATIONAL FOOD POLICY RESEARCH INSTITUTE

$A$ world free of hunger and malnutrition

IFPRI is a CGIAR Research Center

1201 Eye Street, NW, Washington, DC 20005 USA

T. +1-202-862-5600 | F. +1-202-862-5606 | ifpri@cgiar.org | www.ifpri.org

Copyright $\odot 2020$ International Food Policy Research Institute. All rights reserved

Contact ifpri-copyright@cgiar.org for permission to republish.
VISIT A4NH ONLINE www.a4nh.cgiar.org

$\square$ CONTACT A4NH John McDermott A4NH Director j.mcdermott@cgiar.org
TWITTER @A4NH_CGIAR

Wageningen University \& Research leads A4NH's Food Systems for Healthier Diets flagship, with support from the Alliance of Bioversity International and CIAT and IFPRI. To learn more, visit www.a4nh.org 